\title{
Stevens-Johnson Syndrome in a Child on Phenytoin, Exacerbated with Cefixime
}

\author{
Yam Bahadur Roka ${ }^{1}$, Sabrina Shrestha ${ }^{2}$, Narayani Roka ${ }^{3}$ and Mohan Karki ${ }^{4}$ \\ ${ }^{1}$ Department of Neurosurgery, Neuro Cardio and Multispecialty Hospital, Biratnagar, Nepal \\ ${ }^{2}$ Department of Dermatology, Birat Medical College, Biratnagar, Nepal \\ ${ }^{3}$ Department of Ophthalmology, Neuro Cardio and Multispecialty hospital, Biratnagar, Nepal \\ ${ }^{4}$ Department of Neurosurgery, Neuro Cardio and Multispecialty hospital, Biratnagar, Nepal
}

\section{Correspondence:}

Dr Yam Bahadur Roka

Neuro Cardio and Multispecialty Hospital,

Biratnagar, Nepal

E-mail: dryamroka@yahoo.com

DOI: $10.3126 /$ jnps.v39i3.24998

Submitted on: 2019-07-25

Accepted on: 2020-05-03

Acknowledgements: None

Funding: Nil

Conflict of Interest: None declared

Permission from IRB: Yes
To cite this article: Roka YB, Shrestha S, Roka N, Karki M. Stevens Johnson Syndrome in a child on phenytoin, exacerbated with cefixime. J Nepal Paediatr Soc. 2019;39(3):193-6.

\section{ABSTRACT}

Steven Johnson syndrome and toxic epidermal necrolysis are rare but potentially life threatening muco-cutaneous disorders. Their incidence ranges from 1.2 to six per million patient-years for Steven Johnson syndrome and 0.4 to 1.2 per million patientyears for toxic epidermal necrolysis. Drugs are the primary cause for these syndromes in majority cases. They might also be due to infections with Mycoplasma Pneumoniae or Herpes Simplex. The mortality ranges from five to $40 \%$ in these cases. We report a 10-year old girl who presented with history of multiple skin eruptions involving whole body and oral ulceration for five days. She was a known case of seizure disorder on phenytoin and had been prescribed Cefexime for fever. She was managed with intravenous fluids, corticosteroids, opiates, antacids and topical antibiotics. We want to highlight the possibility of Steven Johnson syndrome following the combination of these two drugs.

Key Words: infection; muco-cutaneous disorder; phenytoin; Steven-Johnson syndrome; toxic epidermal necrolysis 


\section{INTRODUCTION}

Steven Johnson syndrome (SJS) and toxic epidermal necrolysis (TEN) are rare but potentially life threatening muco-cutaneous disorders. The incidence of SJS is 1.2 to 6 per million patientyears and of TEN is 0.4 to 1.2 per million patientyears. ${ }^{1}$ Drugs are the cause for these syndromes in the majority of cases. Sometimes, these eruptions may be followed by infections with Mycoplasma Pneumonia or Herpes Simplex. The mortality from these life threatening conditions ranges from $5-40 \%$. Both are characterised by extensive necrosis and erosions of the skin and mucous membrane leading to severe pain, restricted mobility, eat, chew, speak and swallow. They are complicated with secondary infection which can increase both morbidity and mortality. ${ }^{2}$ We report successful management of a rare case of SJS exacerbated with cefixime in a child on Phenytoin for seizure disorder.

\section{CASE REPORT}

A 10-years old girl presented with history of multiple skin eruptions involving whole body for five days. It had progressed to involve the mouth with difficulty in eating or drinking. She was a known case of seizure disorder who had been taking tab Phenytoin $200 \mathrm{mg}$ at night regularly for the past one year. On further questioning it was revealed that she had fever with cough and sore throat for 10 days for which cefixime had been prescribed along with anti-inflammatory medicine. On the third day of taking the new medication she started to have these rashes. On examination she was alert and conscious but in obvious discomfort. There were multiple discrete rashes with asymmetrical involvement and ulcers involving the whole body, scalp, face and all the extremities including the mouth (Figure 1). Features of ulcer bleed with secondary infection were also present. There was no involvement of the gastrointestinal tract or the pulmonary system. She was dehydrated and in extreme pain. Her haematological examination showed raised counts with normal renal and hepatic function tests.

She was managed with intravenous fluids, corticosteroids, opiates, antacids and topical antibiotics. Mouth wash and oral triamcinolone ointment was applied to the ulcers. Phenytoin was tapered over five days and changed over to levetiracetam. Her rashes slowly improved over a week and she was discharged after her rashes had improved. Presently, she has been fine and is on our regular follow up.

\section{DISCUSSION}

SJS is rare and possible fatal cause of mucocutaneous adverse skin reaction usually secondary to drugs or infection and overlapping with TEN. The most common medicines to cause SJS are antiepileptics (Phenytoin, Carbamazepine, Lamotrigine), non-steroidal anti-inflammatory drugs, allopurinol or other antibiotics like aminopenicillins and quinolones, with the incidence of five per million users. ${ }^{3,4}$ Cephalosporin association with SJS is uncommon and the first-generation cephalexin has been reported in several case reports. The first case report was in 1975 in a nine month old infant and since then there have been occasional reports of similar cases.

Phenytoin has a 90\% plasma protein binding property and thus near the therapeutic range even low doses can lead to toxicity. It is also a hepatic enzyme inducer and thus interacts by reducing levels of other drugs. Around $19 \%$ of the patients taking phenytoin can have SJS during the initial two months of therapy. ${ }^{5}$ Cephalosporins has been found to react with lysine and nucleophilic group of

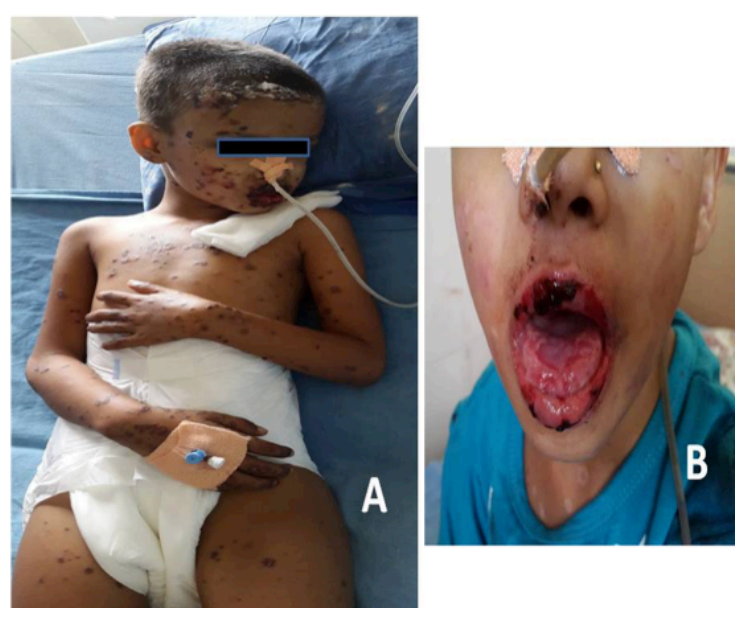

Figure 1. Clinical picture showing the extent of SJS on the body, face and mouth. The scalp, face, trunk and all four extremities are involved (A) and close-up showing the stomatitis and mucocutaneous ulcers (B) 
protein to convert into cephalosporin which is antigenic leading to SJS.6,7

SJS should be differentiated with staphylococcal scalded skin syndrome, impetigo, pemphigus vulgaris and bullous pemphigoid and it is associated with myalgia, fever, mucosal involvement and the upper trunk or even the back. There is difficulty to eat and speak especially with skin blisters and hemorrhagic eruptions around the mouth. The eyes may be involved to cause conjunctivitis. The drug leads to $\mathrm{T}$ lymphocyte mediated cell death, apoptosis and skin detachment. Clinically it manifests as skin rashes with purpuric macular patch with central necrosis in an asymmetric manner in the trunk and limbs. There is no definitive diagnostic method and the diagnosis is made by clinical examination alone. Treatment involves general care to the ulcers, prevention of secondary infection, feeding and nutrition, analgesics and electrolyte and glucose control. Dressing of the wounds with surface antibiotic and use of nonstick coverings are important. Steroids, although controversial, have been used to manage these cases. ${ }^{8}$ The anti-epileptic must be tapered and stopped along with use of other medications and the antibiotic exposure prevented. Another study associated $\mathrm{CYP} 2 \mathrm{C} 9 * 3$ allele with increased incidence of SJS in patients taking Phenytoin and thus could help in screening. ${ }^{9}$

This case represents an interesting clinical finding of the association of SJS aggravated with cefexime use. Although phenytoin itself can cause SJS, it usually occurs within the first three to four months after treatment. Here, our patient was already on phenytoin for a year without any adverse reactions and the short history of fever along with the use of cefexime proves the latter to be a cause SJS. Phenytoin itself is commonly prescribed for seizure disorder as it is effective, cheap and easily available. In developing countries cephalosporins are frequently prescribed for respiratory infections and thus may aggravate SJS in cases taking phenytoin. An understanding of this pathology, proper history of the use of anti-epileptics and keeping an eye for such complication can help to manage these cases effectively.

\section{CONCLUSIONS}

SJS may be associated with a number of medications and is always very dreadful with high incidence of morbidity. In patients on phenytoin it can be exacerbated with cephalosporins and thus caution must be exercised on its simultaneous use.

\section{REFERENCES}

1. Sane SP, Bhatt AD. Stevens-Johnson syndrome and toxic epidermal necrolysis-challenges of recognition and management. J Assoc Physicians India. 2000;48(10):999-1003. PMID: 11200928

2. Murray KM, Camp MS. Cephalexin-induced Stevens-Johnson syndrome. Ann Pharmacother. 1992;26(10):1230-3. DOI: $10.1177 / 106002809202601006$

3. Roujeau JC, Kelly JP, Naldi L, Rzany B, Stern RS, Anderson T, et al. Medication use and the risk of StevensJohnson syndrome or toxic epidermal necrolysis. N Engl J Med. 1995;333:1600-7. DOI: 10.1056/ NEJM199512143332404

4. Roka YB, Roka N, Adhikari HB. Lamotrigine induced severe cutaneous reaction. JNPS. 2012;32(2):172-4. DOI: https://doi.org/10.3126/jnps.v32i2.5608

5. Rzany B, Correia O, Kelly JP, Naldi L, Auquier A, Stern R. Risk of Stevens Johnson syndrome and toxic epidermal necrolysis during first weeks of antiepileptic therapy: a case-control study. Study group of the International case control study on severe cutaneous adverse reactions. Lancet. 1999;353:0-2194. DOI: 10.1016/ s0140-6736(98)05418-x 
6. Prabhu VA, Doddapaneni S, Thunga G, Thiyagu R, Prabhu MM, Naha K. Phenytoin induced Stevens-Johnson syndrome exacerbated by cefepime. J Pharmacol Pharmacother. 2013;4(4):291-3. PMID: 24250210

7. Marco-Del Río J1, Domingo-Chiva E, Cuesta-Montero P, Valladolid-Walsh A, García-Martínez EM. Cefepime Associated with Phenytoin Induced Stevens-Johnson Syndrome. Am J Ther. 2017;24(4):481-4. DOI: 10.1097/MJT. 0000000000000520

8. Hafermann MJ, Barber GR, Dreskin SC, Lindberg GK. Fatal case of Cephalexin-induced toxic epidermal necrolysis. SAGE Open Med Case Rep. 2014;2:2050313X14532250. DOI: 10.1177/2050313X14532250

9. Wu X, Liu W, Zhou W. Association of CYP2C9*3 with Phenytoin-induced Stevens-Johnson syndrome and toxic epidermal necrolysis: A systematic review and meta-analysis. J Clin Pharm Ther. 2018;43(3):408-13. DOI: 10.1111/ jept. 12660 